Georgian Mathematical Journal

Volume 8 (2001), Number 4, 815-822

\title{
A REMARK ON POLYGONAL QUASICONFORMAL MAPS
}

\author{
S. L. KRUSHKAL
}

To Reiner Kühnau on his 65th birthday

\begin{abstract}
Given a quasisymmetric map $h: \hat{\mathbf{R}} \rightarrow \hat{\mathbf{R}}$, let $f_{0}$ be an extremal quasiconformal extension of $h$ onto the upper half-plane $U=\{z \in \mathbb{C}: \Im z>$ $0\}$ whose dilatation $k\left(f_{0}\right)=\inf \left\{k(f): f \mid \partial U=h_{0}\right\}=: k(h)$. Let $k_{n}$ be the minimal dilatation of polygonal quasiconformal maps $f: U \rightarrow U$ satisfying $f\left(x_{j}\right)=h\left(x_{j}\right), j=1,2, \ldots, n$, for any $n$ points of $\hat{\mathbf{R}}$ (vertices of $n$-gons). Already a long time ago, the question was posed whether $k(h)=\sup k_{n}$, where the supremum is taken over all possible $n$-gons of such kind. The answer is obtained (in the negative) for the case of quadrilaterals $(n=4)$.

We show that in the case of pentagons $(n=5)$ the answer is also negative, i.e., there are quasisymmetric $h$ with $k(h)>\sup k_{5}$.
\end{abstract}

2000 Mathematics Subject Classification: Primary: 30C62, 30C75, 30F60. Secondary: 30C35, 30F45, 31A15.

Key words and phrases: Extremal quasiconformal maps, polygon, Grunsky coefficients, Fredholm eigenvalues.

1. Introduction. Any quasisymmetric homeomorphism $h$ of $\hat{\mathbf{R}}=\mathbf{R} \cup\{\infty\}$ onto itself, i.e., such that

$$
M^{-1} \leq \frac{h(x+t)-h(x)}{h(x)-h(x+t)} \leq M
$$

for any $x \in \mathbf{R}$ and $t>0$, with $M=M(h)<\infty$, admits quasiconformal extensions onto the upper half-plane $U=\{z: \Im z>0\}$ (and symmetrical extensions to the lower half-plane $\left.U^{*}=\{z: \Im z<0\}\right)$.

Let $f_{0}$ be an extremal quasiconformal extension of $h$ whose dilatation

$$
k\left(f_{0}\right)=\inf \left\{k(f)=\left\|\partial_{\bar{z}} f / \partial_{z} f\right\|_{\infty}: f \mid \partial U=h\right\}=: k(h) .
$$

Recall that quasiconformal maps are (generalized) homeomorphic solutions of the Beltrami equation $\partial_{\bar{z}} w=\mu(z) \partial_{z} w$ in a domain $D \subset \hat{\mathbf{C}}=\mathbb{C} \cup\{\infty\}$ with $\|\mu\|_{\infty}<1$, where the derivatives $\partial_{\bar{z}}$ and $\partial_{z}$ are distributional and belong locally to $L_{2}$. The function $\mu$ is called the Beltrami coefficient of the map $w$, and the quantity $K(f)=(1+k(w))(1-k(w))$ is its maximal dilatation. The maps with $k(w) \leq k_{0}$ are called $k_{0}$-quasiconformal.

ISSN 1072-947X / \$8.00 / C) Heldermann Verlag www.heldermann.de 
Given a simply connected Jordan domain $D \subset \hat{\mathbf{C}}$, one can regard it as a (topological) polygon $D\left(z_{1}, \ldots, z_{n}\right)$ whose vertices are $n$ distinguished boundary points $z_{1}, \ldots, z_{n}$ ordered in accordance with the orientation of $\partial D$. Quasiconformal maps of polygons moving the vertices into vertices will be called polygonal quasiconformal.

In view of conformal invariance of $q$-quasiconformality, it will suffice for us to deal with the maps of polygons whose domains are either the upper half-plane $U$ or the unit disk $\Delta=\{z:|z|<1\}$.

For any quasisymmetric map $h$, we have $k\left(f_{0}\right)=\lim _{n \rightarrow \infty} k\left(f_{n}\right)$, where $f_{n}$ are extremal polygonal quasiconformal maps of $U\left(x_{1}, \ldots, x_{n}\right)$ onto $U\left(h\left(x_{1}\right), \ldots, h\left(x_{n}\right)\right)$, and the set of vertices $\left\{x_{n}\right\}$ becomes dense on $\mathbf{R}$.

Already a long time ago, the question was posed whether for a fixed $n \geq 4$ (or even for $n \leq N$ ) the equality

$$
k(h)=\sup k\left(f_{n}\right)
$$

is achieved when one allows the vertices to vary on $\hat{\mathbf{R}}$ in all possible ways.

The answer is known only in the case of quadrilaterals $(n=4)$. The distortion of conformal modules $\bmod Q$ of all quadrilaterals $D \subset \Delta$ under a quasiconformal homeomorphism $f: D \rightarrow \hat{\mathbf{C}}$ determines its maximal dilatation $K(f)$, and it was conjectured that the bound

$$
K_{0}(h)=\sup _{Q} \frac{\bmod f_{0}(Q)}{\bmod Q}
$$

where $f_{0}$ is an extremal extension of $h$ and the supremum is taken over all quadrilaterals $Q=U\left(z_{1}, z_{2}, z_{3}, z_{4}\right)$, is the best possible.

This conjecture was disproved by a counterexample of Anderson and Hinkkanen (see [1]) and by Reich [15]; a complete answer for quadrilaterals has been given in [21] (see also [12]).

As for $n \geq 5$, it has been showed by Strebel [19] that equality (1) holds for extremal quasiconformal maps $f_{0}$ without essential boundary points.

The purpose of this note is to show that in the case of pentagons $(n=5)$ the answer is also negative:

Theorem. For each $k \in(0,1)$, there exist quasisymmetric maps $h$ with

$$
k(h)=k>\sup k\left(f_{5}\right),
$$

where the supremum is taken over the extremal polygonal maps of all possible pentagons $U\left(x_{1}, \ldots, x_{5}\right)$.

The proof goes along the lines of [15] and [5]; it involves the fundamental Reich-Strebel inequality for polygonal maps and the Grunsky coefficient inequality for holomorphic functions with quasiconformal extension.

2. The Grunsky coefficients. For convenience, we collect here the main facts concerning the properties of the Grunsky matrix of univalent functions. 
We shall use the following notations. Let $D$ be a hyperbolic simply-connected domain in $\hat{\mathbf{C}}$. We denote by $A_{1}(D)$ the subspace of $L_{1}(D)$ formed by holomorphic functions in $D$, and put

$$
<\mu, \varphi>_{D}=\iint_{D} \mu(z) \varphi(z) d x d y, \quad \mu \in L_{\infty}(D), \varphi \in L_{1}(D) \quad(z=x+i y)
$$

and

$$
A_{1}^{2}(D)=\left\{\varphi \in A_{1}(D): \varphi=\omega^{2}\right\} ;
$$

the last set consists of the integrable holomorphic functions in $D$ with zeros of even order.

The Grunsky univalence criterion [3] says that a $\hat{\mathbf{C}}$-holomorphic function

$$
f(z)=z+a_{0}+a_{1} z^{-1}+\cdots
$$

in a neighborhood of the infinite point $z=\infty$ extends to an injective holomorphic function in the disk

$$
D^{*}=\{z \in \hat{\mathbf{C}}:|z|>1\}
$$

(with the same expansion (2)) if and only if its Grunsky coefficients, defined from the expansion

$$
\log \frac{f(z)-f(\zeta)}{z-\zeta}=-\sum_{m, n=1}^{\infty} c_{m n} z^{-m} \zeta^{-n}, \quad(z, \zeta) \in\left(\Delta^{*}\right)^{2},
$$

satisfy for any $x=\left(x_{1}, x_{2}, \ldots\right) \in l^{2}$ the inequality

$$
\left|\sum_{m, n=1}^{\infty} \sqrt{m n} c_{m n} x_{m} x_{n}\right| \leq\|x\|^{2} \quad\left(\|x\|^{2}=\left(\left.\left|\sum_{1}^{\infty}\right| x_{n}\right|^{2}\right)^{1 / 2}\right) .
$$

It is also known that, for functions (3) having $k$-quasiconformal extensions to $\hat{\mathbf{C}}$, inequality (4) is sharpened as follows:

$$
\kappa(f)=\sup \left\{\left|\sum_{m, n=1}^{\infty} \sqrt{m n} c_{m n} x_{m} x_{n}\right|: x \in l^{2},\|x\|=1\right\} \leq k\|x\|^{2} ;
$$

and on the other hand, any holomorphic function $f$ in $\Delta^{*}$ satisfying (5) is univalent and quasiconformally extendible to $\hat{\mathbf{C}}$ with a dilatation $k^{\prime} \geq k$ (see, e.g., [9] [13]; [8], pp. 82-84).

We shall denote the class of univalent functions in $\Delta^{*}$ of form $(3)$ by $\Sigma$, and let $\Sigma(k)$ consist of $f \in \Sigma$ having $k$-quasiconformal extensions to $\hat{\mathbf{C}}$.

The following statement provides a complete description of the class of functions for which inequality (5) is both necessary and sufficient to belong to $\Sigma(k)$, i.e., the value $\kappa(f)$ coincides with the least (extremal) dilatation $k(f)$ among possible extensions of $f$.

Proposition ([5], [6]). The equality

$$
\kappa(f)=\inf \left\{\|\mu\|_{\infty}: w^{\mu} \mid \Delta^{*}=f\right\}
$$


holds if and only if the function $f$ is the restriction to $\Delta^{*}$ of a quasiconformal self-map $w^{\mu_{0}}$ of $\mathbb{C}$ with the Beltrami coefficient $\mu_{0}$ satisfying the condition

$$
\sup \left|<\mu_{0}, \varphi>_{\Delta}\right|=\left\|\mu_{0}\right\|_{\infty},
$$

where the supremum is taken over holomorphic functions $\varphi \in A_{1}^{2}(\Delta)$ with $\|\varphi\|_{A_{1}(\Delta)}=1$.

Geometrically, this means that the Carathéodory metric on the immersion of the holomorphic disk $\left\{t \mu_{0}: t \in \Delta\right\} \subset L_{\infty}(\Delta)$ into the universal Teichmüller space $\mathbf{T}$ equals the intrinsic Teichmüller-Kobayashi metric of this space.

In particular, for any function $f \in \Sigma(k)$ having $k$-quasiconformal extension onto $\bar{\Delta}$ with the Beltrami coefficient $\mu$ of the form

$$
\mu(z)=k|\varphi(z)| / \varphi(z), \quad \varphi \in A_{1}^{2}(\Delta) \backslash\{0\},
$$

we have the equality

$$
\kappa(f)=k .
$$

If the boundary quasicircle $L=f(\partial \Delta)$ is asymptotically conformal, i.e., satisfies

$$
\max _{z \in L(a, b)} \frac{|a-z|+|z-b|}{|a-b|} \rightarrow 1 \text { as }|a-b| \rightarrow 0,
$$

where $L(a, b)$ is the smaller subarc of $L$ between the points $a, b \in L$, then equality (8) is both necessary and sufficient for $f \in \Sigma(k)$ to have (9) ([7]; see also [6], [11]). In particular, this holds for $C^{1}$-smooth curves.

The properties of asymptotically conformal curves and of related conformal maps are described, for example, in [14].

Using Strebel's frame mapping criterion (see [18], [2]), one can establish that each $f \in \Sigma(k)$ mapping the unit circle $S^{1}=\partial \Delta$ onto an asymptotically conformal curve admits a unique extremal quasiconformal extension to $\bar{\Delta}$ of Teichmüller's type, i.e., whose Beltrami coefficient $\mu_{0}=k|\varphi| / \varphi$ with $\varphi \in A_{1}(\Delta) \backslash\{0\}$ (see [7]). This yields (together with (8) and (9)) that for any $f \in \Sigma(k)$ which maps $\Delta^{*}$ onto a domain with asymptotically conformal boundary and has extremal extension with the Beltrami coefficient is of the form

$$
\mu_{0}=k|\varphi| / \varphi, \quad \varphi \in A_{1}(\Delta) \backslash A_{1}^{2}(\Delta),
$$

(i.e., with $\varphi$ having at least one zero in $\Delta$ of odd order), one obtains the strong inequality $\kappa(f)<k$.

The simplest example of coefficients (10) is given by $\mu_{0}(z)=|z|^{m} / z^{m}$ with odd $m \geq 1$.

3. The proof of the Theorem consists of four stages.

(a) For a given quasisymmetric map $h$, let $f_{0}$ be an extremal quasiconformal extension of $h$ to $U$. Fix a pentagon $U\left(a_{1}, a_{2}, a_{3}, a_{4}, a_{5}\right)$ with the vertices $a_{1}, \ldots, a_{5} \in \hat{\mathbf{R}}$. We can assume that these vertices are ordered by

$$
-\infty<a_{1}<a_{2}<\cdots<a_{5}=\infty .
$$


By the general Teichmüller theorem on extremal quasiconformal maps (see, e.g., [20], [4]), the Beltrami coefficient of the extremal map

$$
f: U\left(a_{1}, \ldots, a_{5}\right) \rightarrow U\left(h\left(a_{1}\right), \ldots, h\left(a_{5}\right)\right)
$$

is of the form

$$
\mu_{f}(z)=k \frac{\left|\varphi_{0}(z)\right|}{\varphi_{0}(z)}
$$

where $k=k(f)$ and $\varphi_{0}$ is a rational function of the form

$$
\varphi_{0}(z)=\sum_{k=1}^{4} \frac{c_{k}}{z-a_{k}} .
$$

Here the numbers $c_{k}$ are real because the quadratic differential $\varphi_{0}(z) d z^{2}$ takes the real values on $\mathbf{R}$; hence

$$
\varphi_{0}(\bar{z})=\overline{\varphi_{0}(z)}
$$

First observe that after composing $h$ with the elements of $\operatorname{PSL}(2, \mathbf{R}$ ) (fractional linear automorphisms of $U$ ), the initial pentagon is transformed conformally to a pentagon $U(0,1, a, b, \infty)$ for which representation (11) takes the most simple form

$$
\varphi_{0}(z)=\frac{\left(c_{1}+c_{2}\right) z-\left(c_{1} b+c_{2} a\right)}{z(z-1)(z-a)(z-b)}
$$

which shows that $\varphi_{0}$ has only one real zero. This yields that the extremal quasiconformal maps of any pentagon $D\left(a_{1}, a_{2}, a_{3}, a_{4}, a_{5}\right)$, whose domain $D$ is bounded by a $C^{1+\alpha}$ smooth curve onto other ones, are determind by holomorphic quadratic differentials

$$
\psi_{0}=\left(\varphi_{0} \circ g\right)\left(g^{\prime}\right)^{2},
$$

where $g$ maps conformally $D\left(a_{1}, a_{2}, a_{3}, a_{4}, a_{5}\right)$ onto $U(0,1, a, b, \infty)$, and each $\psi_{0}$ has only one zero; moreover, this zero is located on the boundary of $D$. Consequently, $\psi_{0}$ does not vanish in $D$, and it is possible to define a singlevalued branch of $\sqrt{\psi_{0}}$ in the whole domain $D$.

When at least two vertices of a pentagon tend to the same limit point, it degenerates into an $n$-gon with $n \leq 4$ vertices.

(b) Now, assuming that equality (1) holds for $h$ with $n=5$, one can select a sequence of pentagons

$$
P_{m}=U\left(a_{1, m}, a_{2, m}, a_{3, m}, a_{4, m}, a_{5, m}\right), \quad m=1,2, \ldots,
$$

and the corresponding extremal quasiconformal maps

$$
f_{m}: P_{m} \rightarrow U\left(h\left(a_{1, m}\right), \ldots, h\left(a_{5, m}\right)\right)
$$

which carry the vertices $a_{j}$ to $h\left(a_{j}\right), j=1, \ldots, 5$, so that

$$
\lim _{m \rightarrow \infty} k\left(f_{m}\right)=k(h)=k\left(f_{0}\right) \text {. }
$$

The Beltrami coefficients of these maps are

$$
\mu_{f_{m}}(z)=k_{m}\left|\varphi_{m}(z)\right| / \varphi_{m}(z), \quad k_{m}=k\left(f_{m}\right),
$$


where each $\varphi_{m}(z)$ is a rational function of form (11).

We now apply the Reich-Strebel inequality for polygons to the extremal Beltrami coefficient $\mu_{0}=\mu_{f_{0}}$ of $h$ and to quadratic differentials $\varphi_{m}$ (see [16], Theorem 7) which yields

$$
\Re \iint_{U} \frac{\mu_{0}(z) \varphi_{m}(z)}{1-\left|\mu_{0}(z)\right|^{2}} d x d y \geq \frac{k_{m}}{1-k_{m}}-\iint_{U}\left|\varphi_{m}(z)\right| \frac{\left|\mu_{0}(z)\right|^{2}}{1-\left|\mu_{0}(z)\right|^{2}} d x d y .
$$

For the maps $f_{0}$ with constant $\left|\mu_{0}(z)\right|$ in $U$ (equal to $k(h)$ ), the last inequality implies

$$
\frac{1}{1-k(h)^{2}} \Re<\mu_{0}, \varphi_{m}>_{U} \geq \frac{k_{m}}{1-k_{m}}-\frac{k(h)}{1-k(h)^{2}} .
$$

Combining this with (13), one obtains

$$
\liminf _{m \rightarrow \infty} \Re<\mu_{0}, \varphi_{m}>_{U} \geq k(h),
$$

which is possible only if

$$
\lim _{m \rightarrow \infty} \Re<\mu_{0}, \varphi_{m}>_{U}=k(h) .
$$

Since $\varphi_{m}(z) \neq 0$ in $U$, it follows from (15) that

$$
\sup _{\varphi \in A_{1}^{2}(U):\|\varphi\|_{A_{1}(U)}=1}\left|\left\langle\mu_{0}, \varphi\right\rangle_{U}\right|=k(h) .
$$

(c) Conjugating $h$ and its extensions by

$$
g: \Delta \rightarrow U, \quad \zeta \mapsto z=i(1+\zeta) /(1-\zeta),
$$

we pass to pentagons $\Delta\left(z_{1}, \ldots, z_{5}\right)$ with the vertices on $S^{1}$ and to quasiconformal automorphisms $f$ of the disk $\Delta$, and extend their Beltrami coefficients onto the whole complex plane $\mathbb{C}$, setting $\mu_{f}(z)=0$ for $|z|>1$. Then the corresponding maps are conformal on $\Delta^{*}$, and we normalize them by means of (3). All this does not change the dilatations.

Denote by $\tilde{h}$ and $\tilde{f}_{0}$ the homeomorphisms corresponding to $h$ and $f_{0}$ via such transforms, respectively. Then

$$
\mu_{\tilde{f}_{0}}(\zeta)=-(\mu \circ g) \frac{(\bar{\zeta}-1)^{2}}{(\zeta-1)^{2}}=: \tilde{\mu}_{0}(\zeta)
$$

and, similar to (16),

$$
\sup _{\tilde{\varphi} \in A_{1}^{2}(\Delta):\|\tilde{\varphi}\|_{A_{1}(\Delta)}=1}\left|<\tilde{\mu}_{0}, \tilde{\varphi}>_{\Delta}\right|=k(\tilde{h}) .
$$

This means, due to Proposition, that

$$
\kappa\left(\tilde{f}_{0}\right)=k\left(\tilde{f}_{0}\right)=k(h) .
$$

(d). Now take quasisymmetric homeomorphisms

$$
h=f^{k|\varphi| / \varphi} \mid \mathbf{R}
$$


with arbitrary $k \in(0,1)$ and $\varphi$ satisfying (10) such that quasicircles $\tilde{h}\left(S^{1}\right)$, determined by $h$ following (c), are asymptotically conformal. Then it follows from the above that all such homeomorphisms satisfy (2). This completes the proof of the Theorem.

4. Remark on the Fredholm eigenvalues. Due to Kühnau-Schiffer's theorem (see [10], [17]), the reciprocal value to the Grunsky constant $\kappa\left(\tilde{f}_{0}\right)$ ) is equal to the least positive Fredholm eigenvalue $\lambda_{L}$ of the quasicircle $L=\tilde{f}_{0}\left(S^{1}\right)$ defined by

$$
\frac{1}{\lambda_{L}}:=\sup \frac{\left|D_{f(\Delta)}(u)-D_{f\left(\Delta^{*}\right)}(u)\right|}{D_{f(\Delta)}(u)+D_{f\left(\Delta^{*}\right)}(u)}, \quad D_{G}(u)=\iint_{G}(\nabla u)^{2} d x d y,
$$

where the supremum is taken over functions $u$ continuous on $\hat{\mathbf{C}}$ and harmonic on $\hat{\mathbf{C}} \backslash L$. In the case of a smooth curve, $\lambda_{L}$ coincides with the least positive eigenvalue of the double-layer potential over $L$.

These eigenvalues produce another obstruction to equality (1) for quadrilaterals and pentagons.

\section{REFERENCES}

1. J. M. Anderson and A. Hinkkanen, Quadrilaterals and extremal quasiconformal extensions. Comment. Math. Helv. 70(1995), 455-474.

2. C. J. EARLE and Li ZHONG, Isometrically embedded polydisks in infinite dimensional Teichmüller spaces. J. Geom. Anal. 9(1999), 51-71.

3. H. GRUnsky, Koeffizientenbediengungen für schlicht abbildende meromorphe Funktionen. Math. Z. 45(1939), 29-61.

4. S. L. KrushKal, Quasiconformal mappings and Riemann surfaces. Wiley, New York, 1979.

5. S. L. Krushkal, On the Grunsky coefficient conditions. Siberian Math. J. 28(1987), $104-110$.

6. S. L. KRushkal, Grunsky coefficient inequalities, Carathéodory metric and extremal quasiconformal mappings. Comment. Math. Helv. 64(1989), 650-660.

7. S. L. Krushkal, On Grunsky conditions, Fredholm eigenvalues and asymptotically conformal curves. Mitteilungen Math. Sem. Giessen 228(1996), 17-23.

8. S. L. Krushkal and R. Kühnau, Quasikonforme Abbildungen - neue Methoden und Anwendungen. (Teubner-Texte zur Math., Bd. 54), Teubner, Leipzig, 1983.

9. R. KÜHNAU, Verzerrungssatze und Koeffizientenbedingungen vom Grunskyschen Typ für quasikonforme Abbildungen. Math. Nachr. 48(1971), 77-105.

10. R. KÜHNAU, Quasikonforme Fortzetzbarkeit, Fredholmsche Eigenwerte und Grunskysche Koeffizientenbedingungen. Ann. Acad. Sci. Fenn. Ser. AI. Math. 7(1982), 383-391.

11. R. KüHNAU, Wann sind die Grunskyschen Koeffizientenbedingungen hinreichend für $Q$ quasikonforme Fortsetzbarkeit? Comment. Math. Helv. 61(1986), 290-307.

12. R. Kühnan, Drei Funktionale eines Quasikreises. Ann. Acad. Sci. Fenn. Ser. AI. Math. 25(2000), 413-415.

13. Chr. Pommerenke, Univalent functions. Vandenhoeck \& Ruprecht, Göttingen, 1975. 
14. Chr. Pommerenke, Boundary behaviour of conformal maps. Springer-Verlag, Berlin, 1992.

15. E. Reich, An approximation condition and extremal quasiconformal extensions. Proc. Amer. Math. Soc. 125(1997), 1479-1481.

16. E. Reich AND K. Strebel, Extremal quasiconformal mappings with given boundary values. Contributions to Analysis (L. V. Ahlfors et al., eds.), 375-391, Academic Press, New York and London, 1974.

17. M. Schiffer, Fredholm eigenvalues and Grunsky matrices. Polon. Math. 39(1981), 149164.

18. K. Strebel, On the existence of extremal Teichmüller mappings. J. Anal. Math. 30(1976), 464-480.

19. K. Strebel, On the dilatation of extremal quasiconformal mappings of polygons. Comment. Math. Helv. 74(1999), 143-149.

20. O. Teichmüller, Extremale quasikonforme Abbildungen und quadratische Differentiale. Abh. Preuss. Akad. Wiss., Math.-Naturw. Kl., 1939 (1940), No. 22, 1-197.

21. S. Wu, Moduli of quadrilaterals and extremal quasiconformal extensions of quasisymmetric functions. Comment. Math. Helv. 72(1997), 593-604.

(Received 02.03.2001)

Author's address:

Research Institute for Mathematical Sciences

Department of Mathematics and Computer Science

Bar-Ilan University, 52900 Ramat Gan

Israel 